the technology. But when the report itself highlighted the transmission of disease from animals to humans as one of the council's major concerns, the probability became certainty. Much of the newspaper, television, and radio coverage of the Nuffield Council report has focused strongly on the need to avoid cross-species infection.

Replacing human tissue with animal cells and organs clearly raises issues that some people will find threatening. Spare-parts surgery, using organs or cells from animals, challenges our notions of humanness, our sense of distinctness from other species. It is easy to find sympathy for those who are repelled by xenotransplantation-even if that repulsion has visceral rather than cerebral roots.

In the face of the "yuk" factor, the scientific, knowledge-based position put forward by authoritative bodies must be both accurate and representative. The Nuffield Council concluded that "the risks associated with the possible transmission of infectious diseases as a consequence of xenotransplantation have not been adequately dealt with. It would not be ethical, therefore, to begin clinical trials of xenotransplantation involving human beings."

The higher risk of disease transmission to humans from primates persuaded the Nuffield Council to favor pigs over primates as sources of transplant organs. Its report points to a macaque monkey form of herpes $B$ virus that causes a rapidly fatal encephalitis in humans. It also draws attention to the similarities between the simian and human immunodeficiency viruses (SIV, HIV): Journalists were left to draw their own conclusions about SIV and AIDS. What was lost in all of this distracting detail, however, was the even greater risk that exists of disease transmission in human-to-human transplantation.

Before recombinant DNA, the major sources of insulin for diabetics were the pancreases from pigs and cattle. Despite the fact that there were several million diabetics taking injections of animal insulin several times daily, there were very few, if any, reported cases of bovine or porcine disease transmission. Insulin, of course, was a purified compound, not a living preparation. However, vigilant readers will also recall that purified proteins extracted originally from human sources- human growth hormone extracted from the pituitary glands of human cadavers, for instance, or antihemophilic factor VIII extracted from blood-have far worse records of disease transmission than does animal insulin (see "Bad blood settlement in Japan," p.410). The simple fact is that human beings usually get their diseases from other humans.

The Nuffield Council report also raised the possibility that the animal organs them- selves would be susceptible to animal disease, especially in the case of transplanted lungs. Very little is known about these risks, says the report, and it advises that would-be organ recipients should be advised of the possibility of infection. Naturally. But then perhaps what animal organ recipients should really be advised of, if they want to steer clear of infection, is to reconsider any plans they might have had for a career in pig-farming. That, one suspects, would not be an untenable burden at least for the 98 percent of us who forsook agriculture for the urban lifestyle a long while ago.

The Nuffield Council report also draws attention to the possibility that prion diseases can pass from one species to another, thereby raising the specter of spongiform encephalopathies. This is simply another distraction. No one is contemplating xenotranplantation from cattle, mink, or cats (although a patient did receive a sheep heart in 1968 and died instantly). Furthermore, as the report itself describes later, the transmission of prion diseases normally occurs by transplantation or by eating infected material. If there are pig prions, avoiding bacon and ham would seem to be as wise a course of action as curtailment of xenotransplantation.

There are, undoubtedly, some very nasty diseases that affect both animals and humans. There are also very many more diseases that are virtually species-specific and highly unlikely under any circumstance to cause any human disease, ever. Furthermore, one could call as witnesses for the defense many animal pathogens whose impact on human beings has been very beneficial. Edward Jenner's original observations on the protection against variola (smallpox) - that exposure to vaccinia (cowpox) conferred to milkmaids-spring to mind. Despite the demise of variola, vaccinia is still finding wide employment-as a recombinant expression host in vivo for a variety of diseaseantigen genes in experimental live vaccine. So is fowlpox virus.

Indeed, there is a general message from vaccinologists to those who are worrying about infection in xenotransplantation. From Louis Pasteur-who produced his first rabies virus by passaging rabies through duck brain cultures-to modern producers of influenza vaccines who still cultivate attenuated viruses in chicken eggs, most experience indicates that growing viruses in the cells or tissues of other species can make the infection agent less pathogenic, less fit for its invasive, disease-causing role, and less likely, therefore, to cause a problem in humans.

Nothing here is meant to indicate that infection is not a problem in xenotransplantation. In immunosuppressed patients, it most certainly is. But to address the problem incompletely is to misrepresent the risk. And passing references to headline-grabbing diseases that are in all likelihood irrelevantEbola gets a mention as well as AIDS and bovine serum encephalitis-distract from the real disease issues. Asilomar and its consequences for public attitudes to recombinant DNA should alert the research community to the dangers of being too "responsible" in the face of uncertainty. If we don't know something, lets just say so and leave it at that.

\section{Ex Novartis ad astra}

The name "Novartis," for the new entity that was Ciba-Geigy and Sandoz, may be pretentious but it has merit, even hidden depth.

The first is that a new name clearly signals a clean beginning and discards the banners to which old loyalties and rivalries might have been directed.

"Novartis" also has a certain style. Not yet elegance, of course. That would be too shocking, at least for those involved in the pharmaceutical industry, who, in their naming of drugs, seem to delight so in the torture and corruption of the language. "Novartis" cannot - in a way that so many merged corporate "famous names" can-be mistaken for an advertising agency or a firm of New York lawyers. A design consultancy? Perhaps.

Novartis has classical roots. It is a reversal and diminution of the Latin "artis nova," meaning "new skill" or "innovation." The intended innovation, no doubt, is internal. But, as with other life science company mergers, there will be implications for smaller innovative companies, too. The recent creations of the lawyerly Glaxo Wellcome (London), Pharmacia and Upjohn (Kalamazoo, MI and Stockholm, Sweden) and Hoechst Marion Roussel (Frankfurt, Germany), for example, have involved substantial job losses. This means that small biotechnology companies may find themselves able to recruit unexpectedly available experienced pharmaceutical executives at prices they can afford. And more adventurous former Ciba or Sandoz employees may turn entrepreneur and seek financial backing to establish their own companies-as did former Burroughs-Wellcome executives in founding Triangle Pharmaceuticals (Research Triangle Park, NC). On the downside, partnering may get somewhat harder. In the midst of introspective postmerger consolidation, smaller companies may find it more difficult to get their pitch across to the new megafirms. And fewer, bigger life science giants means fewer players at the biotechnology buying table. 